\title{
Ultrashort nonautonomous similariton solutions and the cascade tunneling of interacting similaritons
}

\author{
Rongcao Yang ${ }^{1,2,3, *}, \mathrm{Jie}_{\mathrm{Gao}}{ }^{1}$, Heping Jia ${ }^{1}$, Jinping $\operatorname{Tian}^{1,3}$ and J. M. Christian ${ }^{4}$ \\ ${ }^{1}$ School of Physics \& Electronics Engineering, Shanxi University, Taiyuan, 030006, China \\ ${ }^{2}$ Collaborative Innovation Center of Extreme Optics, Shanxi University, Taiyuan, 030006, China \\ ${ }^{3}$ The State Key Laboratory of Integrated Optoelectronics, Chinese Academy of Sciences, Beijing, 100083, China \\ ${ }^{4}$ Joule Physics Laboratory, School of Science, Engineering and Environment, University of Salford, Greater Manchester, UK \\ *Corresponding author: sxdxyrc@sxu.edu.cn
}

\begin{abstract}
Similarity transformation and Hirota bilinearization are deployed to derive exact bright and dark ultrashort one- and two-similariton solutions of a nonautonomous cubic-quintic nonlinear Schrödinger equation. Such wave packets may emerge when group-velocity dispersion and cubic-quintic self-phase modulations are balanced by Raman self-frequency shift in the presence of an external harmonic trap and linear gain or loss. The solutions presented here can be used to investigate the compression, amplification and interaction phenomena associated with bright and dark similaritons in inhomogeneous fiber systems. Furthermore, the dynamics of the characteristic parameters of the similaritons are studied analytically, and similariton stability in a distributed system is tested through extensive computations. As an example application, the tunneling behavior of bright and dark similaritons through cascade dispersion barriers and dispersion wells on an exponential background is investigated, and some interesting novel features are uncovered which are expected to facilitate the control of bright and dark ultrashort similariton in experimental scenarios.
\end{abstract}

Keywords two-similariton, interaction, Raman effect, harmonic potential, cascade tunneling

\section{Introduction}

Optical similaritons originate from the interplay between group-velocity dispersion (GVD), nonlinearity and linear gain/loss in optical fiber lasers [1]. These waves can preserve their overall shapes during propagation by adjusting their amplitudes and widths to accommodate gradual longitudinal variations in system parameters [1-3]. Much attention in the optics literature has been paid to similaritons in fiber lasers [3-7] and amplifiers [8,9], dispersion-decreasing fibers [10-12], and tapered inhomogeneous nonlinear waveguides [13-18]. Generic theoretical models governing the dynamics of similaritons tend to be based on the well-known nonlinear Schrödinger (NLS) equation [1,2]. However, for systems supporting high-intensity ultrashort pulses, higher-order nonlinear effects can come into play such as the host medium's quintic response and also the Raman self-frequency shift. To accommodate these additional contributions to wave propagation, the nonautonomous cubic-quintic nonlinear Schrödinger (C-QNLS) equation is proposed in non-dimensional form [19-21]

$$
i u_{z}+\frac{\beta(z)}{2} u_{t t}+\gamma(z)|u|^{2} u+\alpha(z)|u|^{4} u+\frac{p(z)}{2} t^{2} u+i \delta(z) u\left(|u|^{2}\right)_{t}=i \Gamma(z) u,
$$

where $z$ and $t$ are the normalized propagation distance and the retarded time, respectively, and $u(z, t)$ is the complex slowly-varying envelope of the electric field. The functions $\beta(z), \gamma(z)$ and $\alpha(z)$ modulate the GVD, cubic and quintic nonlinearities, respectively, while $p(z)$ denotes the external harmonic potential, $\delta(z)$ accounts for retarded Raman shifting, and $\Gamma(z)$ parametrizes the gain or loss. 
Equation (1) is of interest across several branches of modern research including plasma physics, Bose-Einstein condensates, and particularly nonlinear fiber optics [19-22]. Simplified versions, where one or more terms are neglected, have been studied previously in the contexts of control, tunneling and compression of bright, dark, kink and anti-kink similaritons $[10,11,19,21]$. But in the most general regime - when the terms modelling quintic nonlinearity, an external potential and the Raman effect are all retained - there arise some key issues that have not yet been addressed in the literature. Fundamentally, there is the central question of solution existence: finding multi-similaritons of nonautonomous systems such as Eq. (1) presents somewhat greater theoretical complexity than in the simpler cases considered to date. And should such waves exist, their stability across a wide parameter space and the tunneling characteristics of two interacting similaritons passing through cascade barriers and wells need to be investigated.

Motivated by these issues, this paper considers Eq. (1) in full and seeks its nonautonomous bright and dark one- and two-similariton solutions. In Section 2, the bright families are derived by deploying similarity transformations while the derivation of dark families requires an additional level of analysis by using Hirota's bilinearization method. The parameter dynamics and the stability discussion of the obtained similaritons are also presented. As a candidate

application, the tunneling features of the novel similaritons passing through dispersion barriers (DBs) and dispersion wells (DWs) are explored in Section 3. Finally, we summarize results and draw conclusions in Section 4.

\section{Ultrashort nonautonomous similariton solutions}

In the absence of the harmonic-potential and Raman-shift terms in Eq. (1), Ref. [23] presented bright one- and twosimilariton solutions for the constant-coefficient C-QNLS equation. Our general analytical approach, then, involves establishing a one-to-one correspondence between Eq. (1) and its constant-coefficient counterpart through similarity transformations. However, to our best of our knowledge, dark similariton solutions for both constant- and variablecoefficient C-QNLS equations remain unknown; moreover, we cannot obtain dark similaritons for Eq. (1) by the similarity transformation technique alone. Rather, dark one- and two-similariton solutions of Eq. (1) will be sought by using Hirota's bilinearization method in combination with a similarity transformation.

\subsection{Bright similariton solutions}

To obtain exact bright similariton solutions for Eq. (1), we begin by introducing the similarity transformation

$$
u(z, t)=\rho(z) \Phi(Z(z), T(z, t)) \exp [i \phi(z, t)]
$$

where $T(z, t)$ is a similarity variable, and $\rho(z)$ and $Z(z)$ represent the amplitude and the effective propagation distance, respectively. By substituting ansatz (2) into Eq. (1), one can arrive at a constant-coefficient C-QNLS equation which also possesses a Raman-shift term (this model is known as the Kundu-Eckhaus equation [20,23]) such that

$$
i \Phi_{Z}+a_{1} \Phi_{T T}+a_{2}|\Phi|^{2} \Phi+a_{3}|\Phi|^{4} \Phi+i a_{4} \Phi \frac{\partial|\Phi|^{2}}{\partial T}=0,
$$

with $a_{1}=\beta(z) T_{t}^{2} / 2 Z_{z}, a_{2}=\gamma(z) \rho^{2} / Z_{z}, a_{3}=\alpha(z) \rho^{4} / Z_{z} \quad a_{4}=\delta(z) T_{t} \rho^{2} / Z_{z}$. Meanwhile, we can obtain the following constraint relations: 


$$
\begin{aligned}
& w_{z z}(z) \beta(z)-w_{z}(z) \beta_{z}(z)-p(z) \beta^{2}(z) w(z)=0, \\
& 2 a_{1} \alpha(z) \exp \left[4 \int_{0}^{z} \Gamma(s) d s\right]-a_{3} \beta(z)=0, \\
& 2 a_{1} \delta(z) \exp \left[2 \int_{0}^{z} \Gamma(s) d s\right]-a_{4} \beta(z)=0,
\end{aligned}
$$

where $w(z)=a_{2} \beta(z) w_{0} \exp \left[2 \int_{0}^{z} \Gamma(s) d s\right] / 2 a_{1} \gamma(z)$. Since we are looking to solve Eq. (1) by first solving Eq. (3), the formulation of the similariton solutions to Eq. (1) will evidently also be bound by constraints (4a)-(4c); these relations inherently capture the $z$-dependence of the coefficients in Eq. (1). After some calculations, it can be shown that the parameters of the similaritons are given by

$$
\begin{gathered}
\rho(z)=\frac{1}{w(z)} \sqrt{\frac{a_{2} \beta(z)}{2 a_{1} \gamma(z)}}, \\
\phi(z, t)=\frac{w_{z}(z)}{2 \beta(z) w(z)} t^{2}-\frac{1}{w(z)} t-\frac{1}{2} \int \frac{\beta(z)}{w^{2}(z)} d z, \\
T=\frac{t-t_{c}(z)}{w(z)}, t_{c}(z)=t_{0}-w(z) \int \frac{\beta(z)}{w^{2}(z)} d z, Z=Z_{0}+\frac{1}{2 a_{1}} \int \frac{\beta(z)}{w^{2}(z)} d z,
\end{gathered}
$$

where $t_{0}, w_{0}$ and $Z_{0}$ denote the initial values of the corresponding parameters at $z=0$, respectively. Without loss of generality, here we set $t_{0}=0, Z_{0}=0$, and $w_{0}=1$. Thus, based on the solutions to Eq. (3) [23], one can derive the bright one- and two-similariton solutions to Eq. (1) by deploying the parameters defined in Eqs. (5a)-(5c) but subject to the additional condition $2 \beta(z) \alpha(z)+\delta^{2}(z)=0$. The bright one-similariton solution satisfying Eq. (1) can now be expressed in the form

$$
u_{1-b r i g h t}(z, t)=\rho(z) \Phi_{1-b r i g h t}(z, t) \exp [i \phi(z, t)]
$$

where

$$
\begin{gathered}
\Phi_{1-b r i g h t}(z, t)=\frac{m_{1}}{2} \operatorname{sech}\left[\frac{\lambda_{1}}{2}+\theta(z, t)\right] \exp \left(-\frac{\lambda_{1}}{2}-\int \frac{2 a_{3}\left|m_{1}\right|^{2} \exp \left[\xi_{1}(z, t)\right]}{a_{4} w(z)\left[1+\exp \left[\xi_{1}(z, t)\right] \exp \left(\lambda_{1}\right)\right]^{2}} d t+i \varphi_{1}(z, t)\right) \\
\theta(z, t)=k_{1 r} \frac{t-t_{c}(z)}{w(z)}-k_{1 i} \int \frac{\beta(z)}{w^{2}(z)} d z \\
\varphi_{1}(z, t)=k_{1 i} \frac{t-t_{c}(z)}{w(z)}+\frac{k_{1 r}^{2}-k_{1 i}^{2}}{2} \int \frac{\beta(z)}{w^{2}(z)} d z \\
\exp \left(\lambda_{1}\right)=\frac{a_{2}}{8 a_{1}} \frac{m_{1} m_{1}^{*}}{k_{1 r}^{2}}, \xi_{1}(z, t)=2 k_{1 r} \frac{t-t_{c}(z)}{w(z)}-2 k_{1 i} \int \frac{\beta(z)}{w^{2}(z)} d z
\end{gathered}
$$

In addition, the bright two-similariton solution can be expressed in the form 


$$
u_{2-b r i g h t}(z, t)=\rho(z) \Phi_{2-b r i g h t}(z, t) \exp [i \phi(z, t)]
$$

where

$$
\begin{aligned}
& \Phi_{2-b r i g h t}(z, t)=\frac{G}{F} \exp \left(-i \int \frac{2 a_{3}}{a_{4} w(z)} \frac{|G|^{2}}{F^{2}} d t\right), \\
& G(z, t)=m_{1} \exp \left(\zeta_{1}\right)+m_{2} \exp \left(\zeta_{2}\right)+\gamma_{1} \exp \left(\xi_{1}+\zeta_{2}\right)+\gamma_{2} \exp \left(\zeta_{1}+\xi_{2}\right) \\
& F(z, t)=1+\exp \left(\zeta_{1}+\zeta_{2}^{*}\right) \exp \left(\lambda_{0}\right)+\exp \left(\zeta_{1}^{*}+\zeta_{2}\right) \exp \left(\lambda_{0}^{*}\right)+\exp \left(\xi_{1}\right) \exp \left(\lambda_{1}\right) \\
& +\exp \left(\xi_{2}\right) \exp \left(\lambda_{2}\right)+\exp \left(\xi_{1}+\xi_{2}\right) \exp (\lambda) \\
& \gamma_{1}=\frac{a_{2}}{8 a_{1}} m_{2}\left|m_{1}\right|^{2} \frac{\left(k_{1 r}-k_{2 r}+i k_{1 i}-i k_{2 i}\right)^{2}}{k_{1 r}^{2}\left(k_{1 r}+k_{2 r}+i k_{2 i}-i k_{1 i}\right)^{2}} \\
& \gamma_{2}=\frac{a_{2}}{8 a_{1}} m_{2}\left|m_{1}\right|^{2} \frac{\left(k_{1 r}-k_{2 r}+i k_{1 i}-i k_{2 i}\right)^{2}}{k_{2 r}^{2}\left(k_{1 r}+k_{2 r}-i k_{2 i}+i k_{1 i}\right)^{2}} \\
& \zeta_{j}(z, t)=\left(k_{j r}+i k_{j i}\right) \frac{t-t_{c}(z)}{w(z)}+i \frac{k_{j r}^{2}-k_{j i}^{2}+i 2 k_{j i}}{2} \int \frac{\beta(z)}{w^{2}(z)} d z \quad(j=1,2), \\
& \xi_{j}(z, t)=2 k_{j r} \frac{t-t_{c}(z)}{w(z)}-2 k_{j i} \int \frac{\beta(z)}{w^{2}(z)} d z \quad(j=1,2), \\
& \exp \left(\lambda_{0}\right)=\frac{a_{2}}{2 a_{1}} \frac{m_{1} m_{2}^{*}}{\left(k_{1 r}+k_{2 r}+i k_{1 i}-i k_{2 i}\right)^{2}}, \exp \left(\lambda_{2}\right)=\frac{a_{2}}{8 a_{1}} \frac{m_{2} m_{2}{ }^{*}}{k_{2 r}^{2}}, \\
& \exp (\lambda)=\frac{a_{2}^{2}}{16 a_{1}^{2}} \frac{\left|m_{1}\right|^{2}\left|m_{2}\right|^{2}\left[\left(k_{1 r}-k_{2 r}\right)^{2}+\left(k_{1 i}-k_{2 i}\right)^{2}\right]}{k_{1 r}^{2} k_{2 r}^{2}\left[\left(k_{1 r}+k_{2 r}\right)^{2}+\left(k_{1 i}-k_{2 i}\right)^{2}\right]} .
\end{aligned}
$$

Here, $m_{j}, k_{j r}$ and $k_{j i}(j=1,2)$ are arbitrary real constants. The two-similariton solution (8) is, in essence, a nonlinear superposition of two one-similariton solutions (6). It can be seen from Eqs. (4) and (5) that the pulse width $w(z)$, the central position (time shift) $t_{c}(z)$, and the amplitude $\rho(z)$ all depend explicitly on $\beta(z), \gamma(z)$ and $p(z)$. Moreover, constraints (4a)-(4c) imply that the one- and two- similariton solutions derived here exist as the result of a complicated interplay between all the intrinsic physical processes captured within Eq. (1) and the external potential.

\subsection{Dark similariton solutions}

In order to seek exact dark similaritons of Eq. (1), we first solve Eq. (3) using Hirota's bilinear method and then generate the desired solutions by applying the inverse similarity transformation obtained from (2). Our analysis begins by assuming the following decomposition for the field $\Phi(z, t)[23,24]$ : 


$$
\Phi(z, t)=\frac{g}{f} \exp \left(i \kappa \int \frac{g g^{*}}{f^{2}} d t\right)
$$

where $\kappa$ is a real constant, $g=g(z, t)$ is a complex function and $f=f(z, t)$ is a real function. After substituting ansatz (10) into Eq. (3), one finds that under the condition $2 a_{3}=-a_{4} \kappa$, or equivalently $2 \alpha(z) \exp \left[2 \int_{0}^{z} \Gamma(s) d s\right]+\kappa \delta(z)=0$, the corresponding bilinear form for Eq. (1) must be

$$
\begin{gathered}
{\left[i 2 Z_{z} D_{Z}+\beta(z) T_{t}^{2} D_{t}^{2}\right](g \mathrm{~g} f)=0,} \\
D_{t}^{2}(f \mathrm{~g} f)=\frac{2 \gamma(z) \rho^{2}(z)}{\beta(z) T_{t}^{2}}|g|^{2},
\end{gathered}
$$

where the bilinear operators $D_{z}$ and $D_{t}$ are defined as [24]

$$
D_{z}^{m} D_{t}^{n}(g \cdot f)=\left.\left(\frac{\partial}{\partial z}-\frac{\partial}{\partial z^{\prime}}\right)^{m}\left(\frac{\partial}{\partial t}-\frac{\partial}{\partial t^{\prime}}\right)^{n} g(z, t) f\left(z^{\prime}, t^{\prime}\right)\right|_{z=z^{\prime}, t t^{\prime}} .
$$

In Hirota's method, $g(z, t)$ and $f(z, t)$ are expanded according to [23]

$$
\begin{aligned}
& g=g_{0}\left(1+g_{1}+g_{2}+g_{3}+\ldots . .\right), \\
& f=1+f_{1}+f_{2}+f_{3}+\ldots \ldots .
\end{aligned}
$$

where $g_{j}(j=0,1,2, \ldots n, \ldots)$ and $f_{k}(k=1,2, \ldots n, \ldots)$ are assumed to be differentiable functions of $z$ and $t$. Theoretically, the $n$-similariton solution may be derived by truncating expansions (13a) and (13b) at the $g_{n}$ and $f_{n}$ terms. For the one-similariton solution, it follows that $g=g_{0}\left(1+g_{1}\right)$ and $f=1+f_{1}$; these expressions are then substituted into bilinear forms (11a) and (11b) and the ensuing hierarchy of equations solved sequentially. After extensive calculations, we arrive at the exact dark one-similariton solution of Eq. (1):

$$
u_{1-d a r k}(z, t)=\rho(z) \Phi_{1-\text { dark }}(z, t) \exp [i \phi(z, t)]
$$

with

$$
\begin{gathered}
\Phi_{1-\text { dark }}(z, t)=\eta \exp [i \omega(z, t)] \frac{1+\left(d_{1}+i r_{1}\right) \exp \left[\Omega_{1}(z, t)\right]}{1+\exp \left[\Omega_{1}(z, t)\right]} \exp [i \kappa \mathfrak{R}(z, t)], \\
\mathfrak{R}(z, t)=\int \frac{\eta \eta^{*}\left\{1+\left(d_{1}+i r_{1}\right) \exp \left[\Omega_{1}(z, t)\right]\right\}\left\{1+\left(d_{1}-i r_{1}\right) \exp \left[\Omega_{1}(z, t)\right]\right\}}{w(z)\left[1+\exp \left[\Omega_{1}(z, t)\right]\right]^{2}} d t, \\
\omega(z, t)=\mu \frac{t-t_{c}(z)}{w(z)}+\frac{b}{2 a_{1}} \int \frac{\beta(z)}{w^{2}(z)} d z, \\
\Omega_{1}(z, t)=h_{1} \frac{t-t_{c}(z)}{w(z)}+\frac{\sigma_{1}}{2 a_{1}} \int \frac{\beta(z)}{w^{2}(z)} d z+s_{1},
\end{gathered}
$$




$$
\begin{gathered}
b=\eta \eta^{*} a_{2}-\mu^{2} a_{1}, d_{1}=\frac{h_{1}^{2} a_{1}+a_{2} \eta \eta^{*}}{a_{2} \eta \eta^{*}}, r_{1}= \pm \frac{\sqrt{-h_{1}^{4} a_{1}^{2}-2 h_{1}^{2} \eta \eta^{*} a_{1} a_{2}}}{a_{2} \eta \eta^{*}} \\
\sigma_{1}=\frac{h_{1} a_{1}\left(2 \mu-2 \mu d_{1}-h_{1} r_{1}+i h_{1}+i d_{1} h_{1}-2 i \mu r_{1}\right)}{d_{1}+i r_{1}-1} .
\end{gathered}
$$

To tackle the two-similariton case, expansions (13a) and (13b) are truncated at $g=g_{0}\left(1+g_{1}+g_{2}\right)$ and $f=1+f_{1}+f_{2}$. Then, following a similar lengthy analytical procedure, we obtain the desired exact dark two-similariton solution of Eq. (1):

$$
u_{2-d a r k}(z, t)=\rho(z) \Phi_{2-d a r k}(z, t) \exp [i \phi(z, t)]
$$

with

$$
\begin{gathered}
\Phi_{2-d a r k}(z, t)=\frac{\eta \exp [i \omega(z, t)]\left(1+g_{1}+g_{2}\right)}{1+f_{1}+f_{2}} \exp \left[i \kappa \int \frac{\eta \eta^{*}\left|1+g_{1}+g_{2}\right|^{2}}{w(z)\left(1+f_{1}+f_{2}\right)^{2}} d t\right] \\
g_{1}=\left(d_{1}+i r_{1}\right) \exp \left[\Omega_{1}(z, t)\right]+\left(d_{2}+i r_{2}\right) \exp \left[\Omega_{2}(z, t)\right], f_{1}=\exp \left[\Omega_{1}(z, t)\right]+\exp \left[\Omega_{2}(z, t)\right], \\
g_{2}=\tau_{12}\left(d_{1}+i r_{1}\right)\left(d_{2}+i r_{2}\right) \exp \left[\Omega_{1}(z, t)+\Omega_{2}(z, t)\right], f_{2}=\tau_{12} \exp \left[\Omega_{1}(z, t)+\Omega_{2}(z, t)\right], \\
\Omega_{j}(z, t)=h_{j} \frac{t-t_{c}(z)}{w(z)}+\frac{\sigma_{j}}{2 a_{1}} \int \frac{\beta(z)}{w^{2}(z)} d z+s_{j} \quad(j=1,2), \\
d_{1}= \pm \frac{h_{1}^{2} a_{1}+a_{2} \eta \eta^{*}}{a_{2} \eta \eta^{*}}, d_{2}=\frac{h_{2}^{2} a_{1}+a_{2} \eta \eta^{*}}{a_{2} \eta \eta^{*}} \\
\sigma_{j}=\frac{\sqrt{-h_{1}^{4} a_{1}^{2}-2 h_{1}^{2} \eta \eta^{*} a_{1} a_{2}}}{a_{2} \eta \eta^{*}}, r_{2}= \pm \frac{\sqrt{-h_{2}^{4} a_{1}^{2}-2 h_{2}^{2} \eta \eta^{*} a_{1} a_{2}}}{a_{2} \eta \eta^{*}} \\
r_{j}\left(2 \mu d_{j}-h_{j} r_{j}+i h_{j}+i d_{j} h_{j}-2 i \mu r_{j}\right) \\
d_{j}+i r_{j}-1
\end{gathered}
$$

Here, $\eta$ is a complex constant while $\mu, b, d_{j}, h_{j}, r_{j}, \sigma_{j}(j=1,2)$ are real constants. From inspection of the two square-root expressions in Eq. (17f), one can deduce the constraint ranges of $h_{1}, h_{2}, a_{1}$ and $a_{2}$ to be

$$
h_{1}^{2}<-2 \eta \eta^{*} a_{2} / a_{1} \text { and } h_{2}^{2}<-2 \eta \eta^{*} a_{2} / a_{1}, a_{1} a_{2}<0 .
$$

Since the same similarity transformation (2) has been retained in both our analyses [converting (variable-coefficient) Eq. (1) into (constant-coefficient) Eq. (3)], constraint relations (4a)-(4c) derived for bright similaritons must also hold 
true for dark similaritons. Thus dark similaritons, like their bright counterparts, exist as a result of the balance between the effects of GVD, cubic and quintic nonlinearities, the Raman shift, and the external potential.

\subsection{Properties of similariton solutions}

It can be seen from inspection of solutions (6), (8), (14) and (16) that the width, amplitude, central position (time shift) and chirp of similaritons are described by the functions $w(z), \rho(z), t_{c}(z)$ and $C(z)$, respectively. All four of those characteristic parameters depend directly upon $\beta(z)$ and $\gamma(z)$. Here we consider the evolution of the characteristic parameters of bright and dark similaritons in a typical exponentially-distributed system defined by

$$
\beta(z)=\beta_{0} \exp \left(-\beta_{1} z\right), \quad \gamma(z)=\gamma_{0} \exp \left(-\gamma_{1} z\right)
$$

where the pairs of constants $\left(\beta_{0}, \beta_{1}\right)$ and $\left(\gamma_{0}, \gamma_{1}\right)$ parameterize the GVD and cubic-nonlinearity profiles in the distance $z$, respectively. For this system, one can expect similartons to exist when the external potential, as dictated by Eq. (4a), is given by $p(z)=\frac{\gamma_{1}\left(\gamma_{1}-\beta_{1}\right)}{\beta_{0} \exp \left(-\beta_{1} z\right)}$. It follows that

$$
\begin{gathered}
w(z)=\frac{a_{2} \beta_{0} \exp \left[\left(\gamma_{1}-\beta_{1}\right) z\right]}{2 a_{1} \gamma_{0}}, \\
\rho(z)=\sqrt{\frac{2 a_{1} \gamma_{0}}{a_{2} \beta_{0}}} \exp \left[\left(\beta_{1}-\gamma_{1}\right) z / 2\right], \\
t_{c}(z)=\frac{2 a_{1} \gamma_{0} \exp \left(-\gamma_{1} z\right)}{a_{2}\left(2 \gamma_{1}-\beta_{1}\right)}, \\
C(z)=\frac{\beta_{1}-\gamma_{1}}{\beta_{0} \exp \left(-\beta_{1} z\right)} .
\end{gathered}
$$

From inspection of Eqs. (20a)-(20d), it is clear that the characteristic parameters depend explicitly on the propagation distance and also on the GVD and cubic-nonlinearity distributions. In particular, the amplitude $\rho(z)$ and width $w(z)$ depend upon $\beta_{1}-\gamma_{1}$ and there are hence three different cases to consider. (i) When $\beta_{1}=\gamma_{1}$, the width and amplitude of
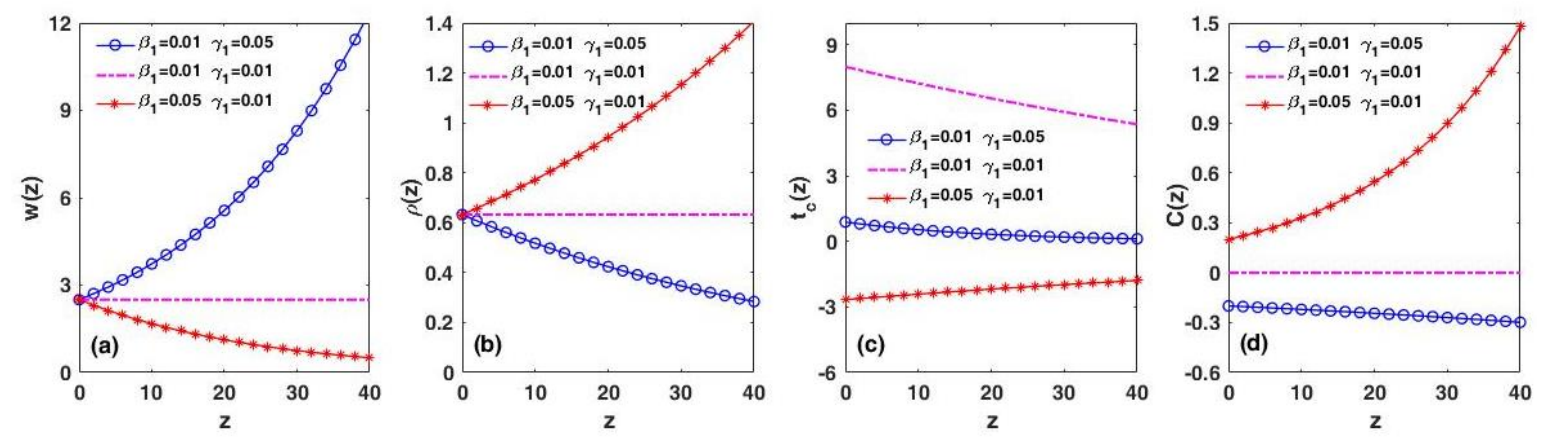

Fig.1. The dynamics of (a) pulse width $w(z)$, (b) amplitude $\rho(z)$, (c) time shift $t_{c}(z)$ and (d) linear chirp $C(z)$ of the similaritons for different dispersion and nonlinearity parameters. Here the parameters are $\beta_{0}=0.2, \gamma_{0}=0.04, a_{1}=1, a_{2}=1$. 
the similaritons are both invariant throughout propagation (which is reminiscent of soliton behavior). (ii) For $\beta_{1}>\gamma_{1}$, the width decreases exponentially with $z$ while the amplitude increases commensurately (this result indicates that pulse compression can be achieved). (iii) For $\beta_{1}<\gamma_{1}$, the opposite features occur (the width increases exponentially and the amplitude decays). Variations in width and amplitude are shown in Figs. 1(a) and 1(b), respectively, with the corresponding time shifts $t_{c}(z)$ and chirps $C(z)$ shown in Figs. 1(c) and 1(d). From solutions (6) and (14), it can be seen that bright and dark one-similaritons have the same linear chirp given in Eq. (20d), but they exhibit different time shifts, denoted by $T_{\text {bright }}(z)$ and $T_{\text {dark }}(z)$, as follows:

$$
\begin{gathered}
T_{\text {bright }}(z)=\left(1-\frac{k_{1 i}}{k_{1 r}}\right) t_{c}(z)-\frac{\lambda_{1} a_{2} \beta_{0}}{4 k_{1 r} a_{1} \gamma_{0}} \exp \left[\left(\gamma_{1}-\beta_{1}\right) z\right], \\
T_{\text {dark }}(z)=\left(1+\frac{\sigma_{1}}{2 a_{1} h_{1}}\right) t_{c}(z) .
\end{gathered}
$$

Figures 2 shows the evolution of bright and dark similaritons with invariant and amplified intensities; the chosen parameters are $m_{1}=1+i, k_{1}=1, a_{1}=1, a_{2}=1, \beta_{0}=0.2, \gamma_{0}=0.05$ for the bright similariton and $\alpha=1+i, \mu=1, h_{1}=1$, $a_{1}=0.55, a_{2}=-0.15, \beta_{0}=0.2, \gamma_{0}=-0.02$ for the dark similariton. When $\beta_{1}=\gamma_{1}$, both waves retain an invariant profile
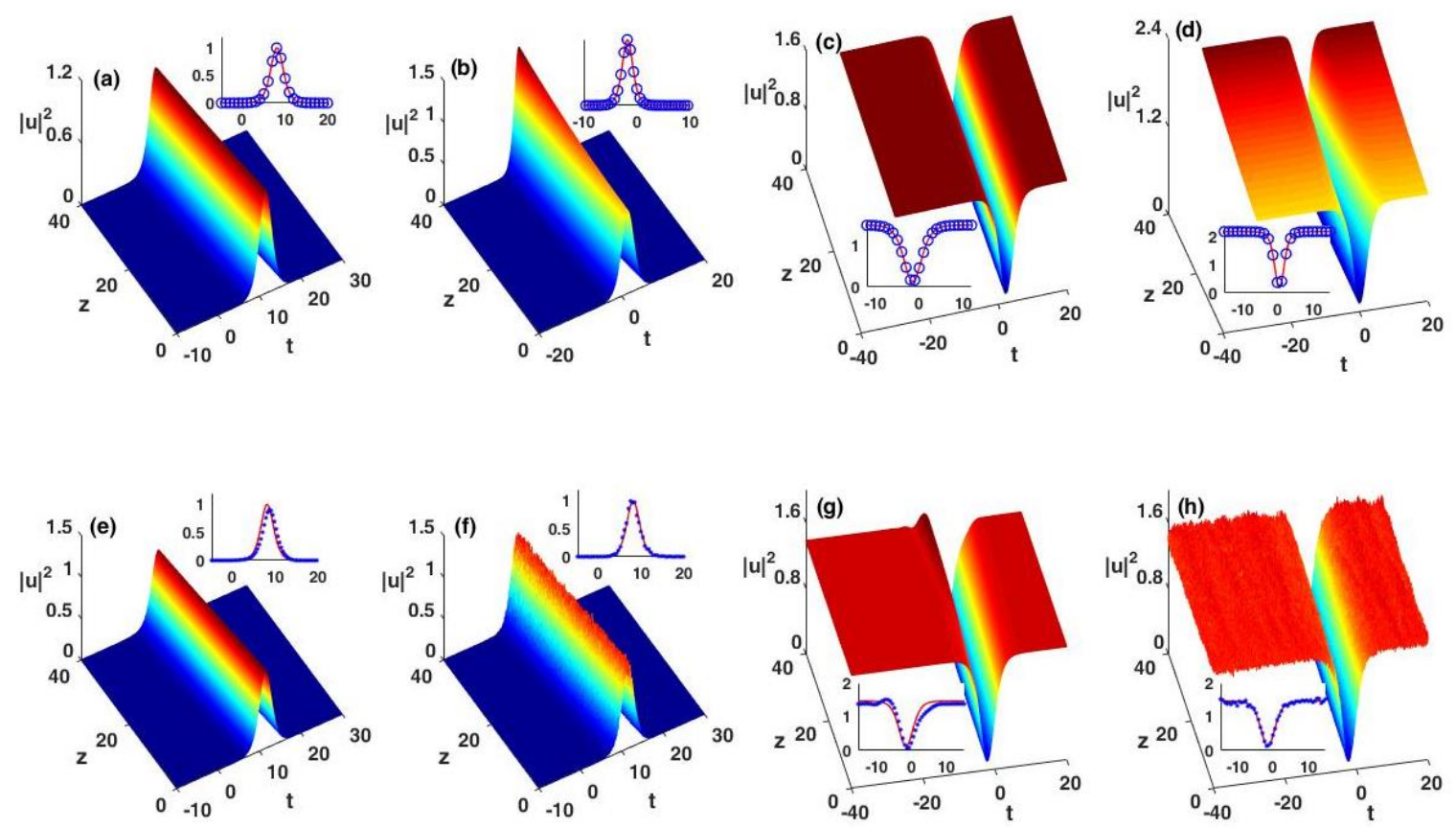

Fig.2. The propagation of bright similariton (6) and dark similariton (14) with the parameters (a) and (c) $\beta_{1}=\gamma_{1}=0.01$; (b) and (d) $\beta_{1}=0.02, \gamma_{1}=0.01$. The other parameters are specified in the text. The numerical evolution of the similaritons under amplitude fluctuation $[(e)$ and (g)] and perturbation of white noise [(f) and (h)], where the parameters are the same as in (a) and (c), correspondingly. The insets are the comparison of numerical [blue circle (dot) lines] and exact profiles (red solid lines) at $\mathrm{z}=40$.

[panes 2(a) and 2(c)] while for $\beta_{1}>\gamma_{1}$ they are compressed and amplified upon propagation [panes 2(b) and 2(d)]. The properties of these bright and dark similaritons predicted from analysis have been verified by direct numerical 
integration of Eq. (1), as shown in the insets of Figs. 2(a)-(d), where simulation results agree extremely well with the exact solutions after propagating a distance of 40 dispersion lengths. Furthermore, we have numerically investigated the stability of bright and dark similaritons against background fluctuations through two distinct classes of perturbed initial-value problems: firstly, by distorting the injected pulse shapes directly (where the similariton initial condition has only $95 \%$ of the amplitude required by the exact solution) and, secondly, by adding a $10 \%$ level of a Gaussian white noise to the initial condition. From the evolution of the similaritons shown in Figs. 2(e)-2(h), we infer that both bright and dark similaritons can be robust under finite perturbations.

Solutions (8) and (16) can be used to study the interaction and tunneling properties of bright and dark two-similaritons in inhomogeneous systems that include, simultaneously, the higher-order effects of quintic nonlinearity, Raman shifting, and an external harmonic trap. As an example application, we now investigate cascade tunneling of twosimilaritons in systems comprising $\operatorname{sech}^{2}$-shaped DBs or DWs. Some interesting features emerge that have, to the best of our knowledge, not been reported in the literature.

\section{The cascade tunneling effects of the interacting similaritons}

The phenomenon of soliton tunneling, first predicted by Newell [25] over four decades ago, has been the subject of intense research in a wide range of nonlinear systems [26-32]. For completeness, we now highlight some key results in the tunneling literature. Serkin et al. [26] considered soliton fission reaction in organic thin films, while G. Y. Yang (G. Y.) et al. [28] investigated the cascade compression of solitons in dispersion-decreasing fiber with nonlinear multibarriers. R. C. Yang et al. [29] studied the tunneling, compression and splitting of spatial solitons in an optical lattice [29], Zhang et al. have demonstrated the controllable compression of spatial solitons passing through nonlinear (diffractive) potential barriers (wells) [30], and Marest et al. observed the longitudinal soliton tunneling process in an axially inhomogeneous fiber [31]. More recently, the tunneling aspects of nonautonomous solitons and similaritons have been investigated from different viewpoints [33-38]. Wang et al. considered similaritons crossing nonlinear barriers with a range of heights [33], Dai et al. investigated bright and dark similariton tunneling based on coupled NLS equations in a birefringent fiber [34], Jia et al. reported the spectral characteristics of chirped nonautonomous soliton through periodic barriers (wells) [35], and Mani Rajan et al. studied nonautonomous solitons and similaritons in tapered graded-index nonlinear waveguides in the context of soliton compression and management [36-38].

To date, few works have addressed the cascade tunneling effects of interacting multi-similaritons in inhomogeneous fiber systems when all the higher-order terms in Eq. (1) are retained. As an application of similaritons (8) and (16), we focus on bright and dark two-similaritons for two different interaction regimes. As our solutions exist only under the balance of GVD, cubic and quintic nonlinearity, Raman shifting and harmonic trapping, their tunneling characteristics in the presence of cascade DBs or DWs might reasonably be expected to exhibit novel features.

We consider a distributed fiber system with cascade DBs or DWs on an exponential background [28]:

$$
\beta(z)=\beta_{0}\left[\exp \left(-\beta_{1} z\right)+\beta_{2} \sum_{j=1}^{n} \operatorname{sech}^{2}\left[v\left(z-z_{0 j}\right)\right],\right.
$$




$$
\gamma(z)=\gamma_{0} \exp \left(-\gamma_{1} z\right)
$$

The summation in Eq. (22a) prescribes an array of $n$ identical $\operatorname{sech}^{2}$-shaped dispersion profiles with inverse width $v$ centered on positions $z=z_{0 j}\left(j=1,2,3, \ldots, n\right.$ ). These constituents correspond to DBs of height $\beta_{2}$ (when $\beta_{2}>0$ ) or to DWs of depth $\left|\beta_{2}\right|$ (when $\beta_{2}<0$ ). In this distributed system (22), the harmonic-potential coefficient $p(z)$ can then be determined from constraint relation (4a). In the special case of no barriers or wells (i.e., $\beta_{2}=0$ ), system (22) reduces to system (19). For that purely-exponential regime, two different interaction scenarios are shown in Fig. 3 [parameters: $\beta_{1}=0.01, \gamma_{1}=0.01$, (a) $\beta_{0}=0.1, \gamma_{0}=0.02, m_{1}=0.05, m_{2}=0.05, k_{1}=1.8-0.95 i, k_{2}=-1.8+0.95 i, a_{1}=0.65$ and $a_{2}=0.15$; (b) $\beta_{0}=0.1, \gamma_{0}=-0.2, \mu=1, h_{2}=-1, s_{1}=2.95, \eta=0.1+i, s_{2}=2.5, h_{1}=0.88, a_{1}=1$ and $a_{2}=-0.55$; (c) $\beta_{0}=0.2, \gamma_{0}=0.1, m_{1}=1+i, m_{2}=1+i, k_{1}=1, k_{2}=-1.1, a_{1}=1$ and $a_{2}=1$; (d) $\beta_{0}=0.2, \gamma_{0}=-0.01, \mu=1, h_{2}=-1$, $s_{1}=2.95, \eta=1+i, s_{2}=0, h_{1}=1, a_{1}=0.55$ and $\left.a_{2}=-0.15\right]$. Both bright and dark two-similaritons preserve their initial velocities after an elastic collision [panes 3(a) and 3(b)] or in the absence of a collision they may propagate with same velocity forming 'parallel stripes' in the $(t, z)$ plane [see panes 3(c) and 3(d)]. The interaction of the similaritons can thus be controlled by selecting appropriate system and solution parameters.
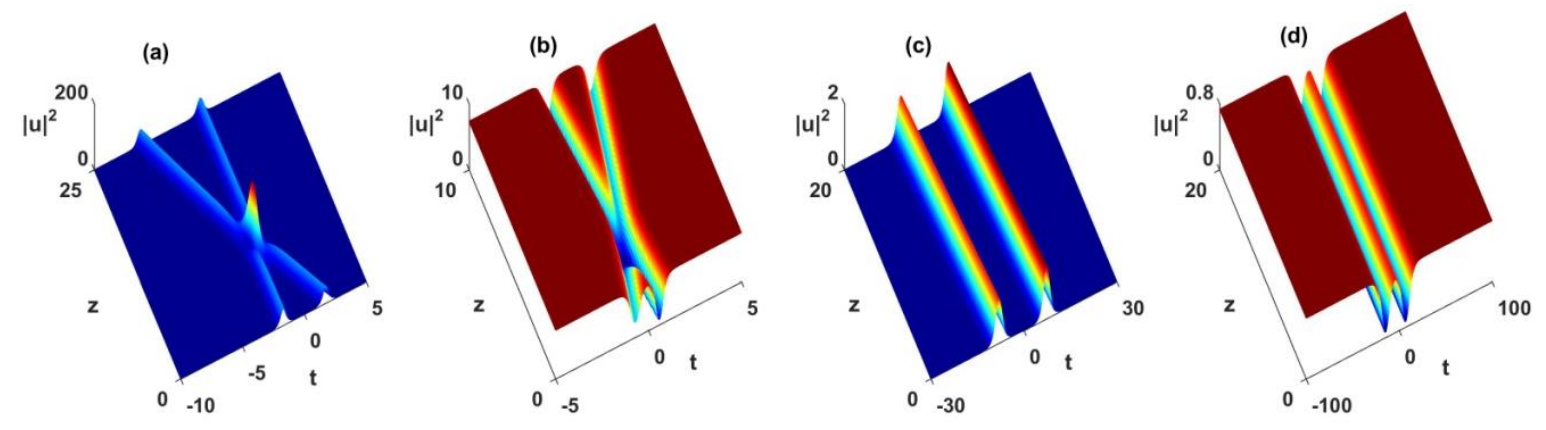

Fig. 3. The interaction scenarios of bright and dark two-similaritons in system (22) with $\beta_{2}=0$. (a) $\beta_{0}=0.1, \gamma_{0}=0.02$; (b) $\beta_{0}=0.1$,

$$
\gamma_{0}=-0.2 ; \text { (c) } \beta_{0}=0.2, \gamma_{0}=0.1 ; \text { (d) } \beta_{0}=0.2, \gamma_{0}=-0.01 \text {. }
$$

\subsection{The tunneling of two parallel similaritons}

We now consider the more general case of system (22), when $\beta_{2} \neq 0$. Figure 4 presents the tunneling behavior of the parallel transmission of bright and dark two-similaritons (8) and (16) passing through the cascade DBs and DWs for $n=3$. On the one hand, it can be seen that as two parallel (i.e., equal-velocity) bright similaritons pass through DBs, they tend to repel each other [repulsion is accompanied by decaying amplitudes and broadening pulse widths; see pane 4(a1)]. On the other hand, the same waves passing through DWs tend to attract each other [attraction is accompanied by pulse compression and the forming of two distinct peaks; see pane 4(b1)]. In a similar manner, besides the tunneling features of the amplitude and pulse width, two parallel dark similaritons also repel each other when passing through DBs and attract each other when passing through DWs [panes 4(c1) and 4(d1), respectively]. Meanwhile, the background wave of the two dark similaritons deforms into valley across each DB and into a ridge across each DW. 
Corresponding to Figs. 4(a1) -4(d1), Figs. 4(a2)-4(d2) depict the pulse distributions of bright and dark two-similaritons at different transmission positions. Both solution families can recover their original shapes after passing through the cascade DBs and DWs. Moreover, when traversing these obstructions, the pulse shapes are cascade compressed and the background-wave deformation into valleys and ridges becomes more pronounced. This implies that one may control the interaction of two-similariton and achieve the desired compression factor with careful design of cascade DBs and DWs.
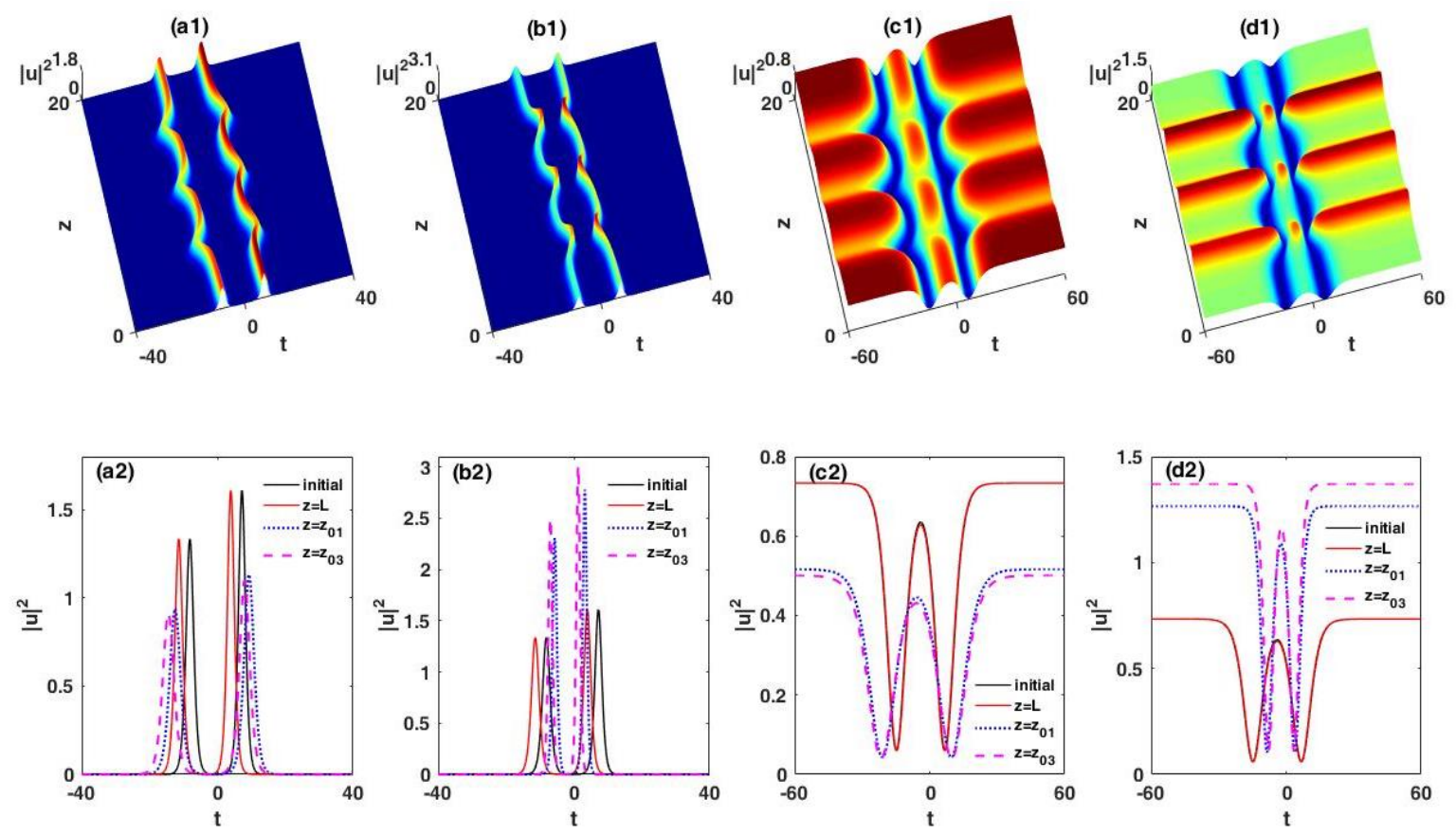

Fig. 4. The tunneling behavior of two parallel similaritons passing through the cascade DBs with $\beta_{2}=0.4$ and DWs with $\beta_{2}=-0.4$. Bright similaritons through (a1) DBs and (b1) DWs; Dark similaritons through (c1) DBs and (d1) DWs, respectively. (a2)-(d2) are the pulse distributions at different transmission positions with $z_{01}=5, z_{03}=10$ and $L=20$, corresponding to (a1)-(d1), respectively. The other parameters are the same as in Fig. 3.

\subsection{The tunneling of two-similaritons in elastic collision}

Figure 5 displays the tunneling behavior of bright and dark two-similaritons (8) and (16) in an elastic collision passing through cascade DBs and DWs given in (22) with $n=4$. Both bright and dark two-similaritons undergo mutual repulsion or mutual attraction depending upon whether the DBs are positioned before or after the collision site [panes 5(a1) and 5(ac)]. When these similaritons tunnel through cascade DWs, the repulsion-attraction features are clearly reversed [panes 5(b1) and 5(d1)]. Comparing panes 5(a1)-5(d1), it can be seen that the similariton with slower velocity accelerates abruptly while the other one with faster velocity decelerates abruptly when passing through cascade DBs [panes 5(a1) and 5(c1)], which can result in repulsions before and attractions after the collision. When both bright and dark similaritons tunnel through the cascade DWs [panes 5(a1) and 5(c1)], they follow the reverse rules that results in repulsions before and attractions after the collision. As in the case of parallel transmission (see Fig. 4), both bright and 
dark two-similaritons undergoing an elastic collision can recover their initial shapes after passing through DBs and DWs. Moreover, both bright and dark two-similaritons can be either broadened or compressed during cascade tunneling which is evident from the corresponding pulse distributions in time [panes 5(a2)-5(d2)]. These results imply that the tunneling characteristics of bright and dark two-similaritons provide a way to realize the compression, amplification and interaction control of these novel families of nonlinear waves.
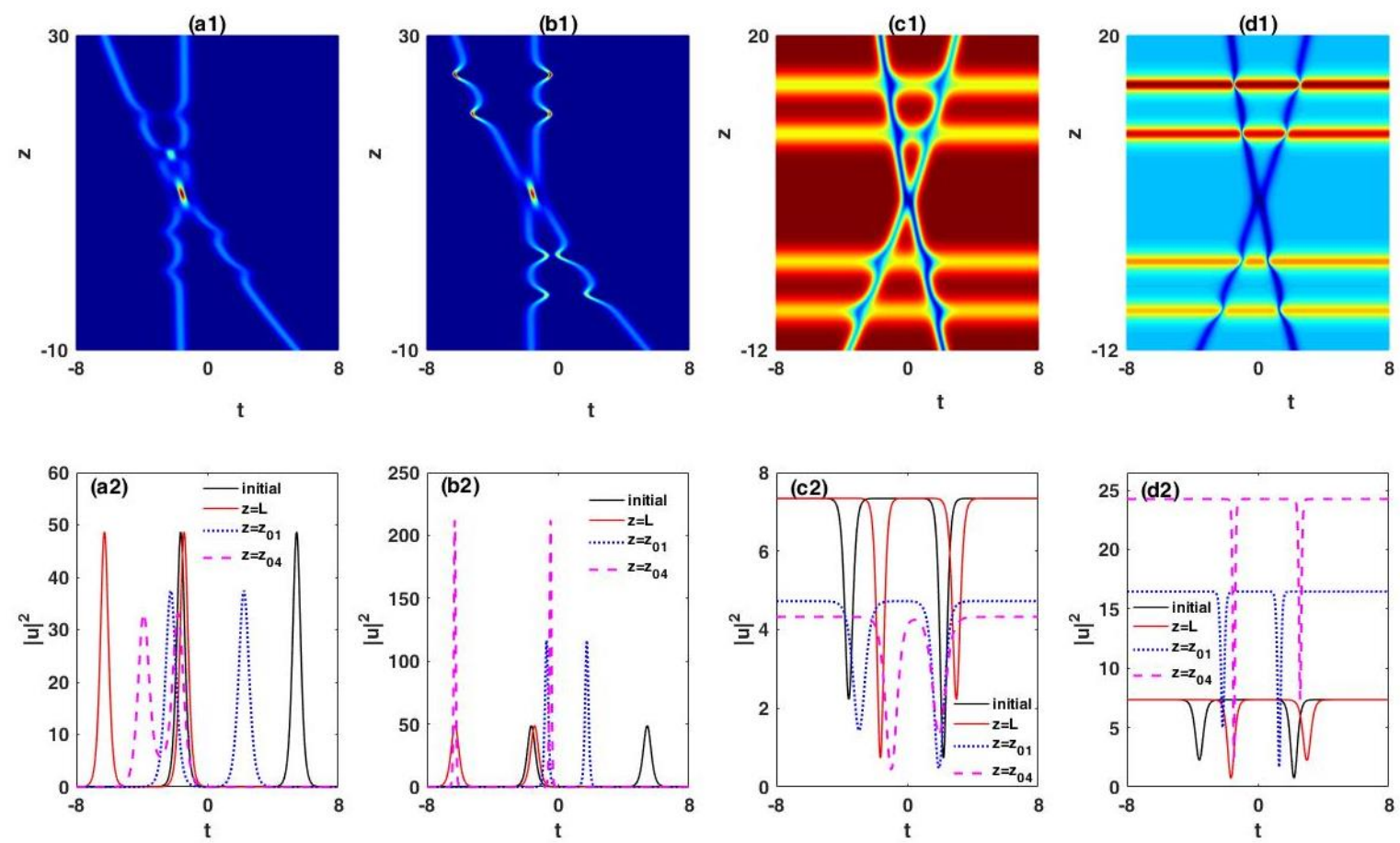

Fig. 5. The tunneling behavior of bright and dark two-similaritons in elastic collision passing through cascade DBs and DWs. Bright similaritons through (a1) DBs with $\beta_{2}=0.3$ and (b1) DWs with $\beta_{2}=-0.6$. Dark similaritons through (c1) DBs with $\beta_{2}=0.6$ and (d1) DWs with $\beta_{2}=-0.6$, respectively. Panes (a2)-(d2) are the corresponding pulse distributions at different transmission positions with (a2) $z_{01}=0$, $z_{04}=20, L=30$; (b2) $z_{01}=-3, z_{04}=25, L=30$; (c2) and (d2) $z_{01}=-8, z_{04}=15, L=20$, respectively. The other parameters are the same as in Fig.3.

\section{Conclusions}

We have derived exact bright and dark ultrashort one- and two-similariton solutions for a variable-coefficient CQNLS equation that accommodates, for the first time, quintic nonlinearity, the Raman self-frequency shift, and an external harmonic potential. The key mathematical techniques deployed throughout have been similarity transformations in combination with Hirota bilinearization. Crucially, these novel solutions can be used to predict the compression, amplification and interaction properties of bright and dark similaritons in generic inhomogeneous systems. We have studied analytically the variations in the characteristic parameters of the similaritons, and numerically investigated their stability in an exponentially-distributed system. Our results show that the compression and amplification of similaritons can be realized and controlled by choosing appropriate system parameters. Moreover, 
both bright and dark similaritons appear to be stable against finite perturbations such as amplitude fluctuations and the addition of white noise.

Tunneling phenomena exhibited by interacting similaritons passing through cascade DBs and DWs have been studied in some detail. It was found that when tunneling through localized dispersive obstructions (in addition to smooth changes in amplitude and width), bright and dark similaritons can exhibit very similar features. Two parallel similaritons (i.e., with equal velocities) repel each other at the position of a DB and attract each other at the position of a DW. While two-similaritons undergoing an elastic collision exhibit mutual repulsion when a DB is positioned before the collision site, and mutual attraction when a DB is positioned after the collision site; the reverse is true when the DBs are replaced with DWs. These results reveal that the tunneling behavior of bright and dark two-similaritons is potentially useful for realizing the compression, amplification and interaction control of multi-similariton waves.

Funding: This work was supported by the National Natural Science Foundation of China (NSFC) [grant number 61775126]; Natural Science Foundation of Shanxi Province [grant numbers 201801D221164, 201801D121119]; Opened Fund of the State Key Laboratory of Integrated Optoelectronics (IOSKL2019KF16).

\section{References:}

[1] J. M. Dudley, C. Finot, D. J. Richardson, G. Millot, Self-similarity in ultrafast nonlinear optics, Nat. Phys. 3 (2007) $597-603$.

[2] V. I. Kruglov, A. C. Peacock, J. D. Harvey, Self-similar propagation of parabolic pulses in normal-dispersion fiber amplifiers, Opt. Soc. Am. B 19 (2002) 461-469.

[3] B. Oktem, C. U. Ilday, F. Ömer, Soliton-similariton fibre laser, Nat. Photonics 4 (2010) 307-311.

[4] Y. X. Tang, Z. W.Liu, W. Fu, F. W. Wise, Self-similar pulse evolution in a fiber laser with a comb-like dispersion-decreasing fiber, Opt. Lett. 41 (2016) 2290-2293.

[5] X. Li, S. M. Zhang, Z. J. Yang, Optimal design of similariton fiber lasers without gain-bandwidth limitation, Opt. Express 25 (2017) 1841018420

[6] L. M. Zhao, D. J. Li, L. Li, X. Wang, Y. Geng, D. Y. Shen, L. Su, Route to Larger Pulse Energy in Ultrafast Fiber Lasers, IEEE JSTQE 24 (2018) 8800409.

[7] Y. F. Song, X. J. Shi, C. F. Wu, D. Y. Tang, H. Zhang, Recent progress of study on optical solitons in fiber lasers, Appl. Phys. Rev. 6 (2019) 021313.

[8] T. S. Raju, Self-similar propagation in a graded-index nonlinear-fiber amplifier with an external source, Phys. Rev. A 81 (2010) 043820.

[9] Y. Liu, D. P. Luo, C. Wang, Z. W. Zhu, W. X. Li, Application of nonlinear pulse shaping of femtosecond pulse generation in a fiber amplifier at $500 \mathrm{MHz}$ repetition rate, Laser Phys. 28 (2018) 035106.

[10] J. D. He, J. F. Zhang, Self-similar optical pulses tunneling in cubic-quintic nonlinear media with distributed coefficients, J. Phys. A: Math. Theor. 44 (2011) 205203.

[11] C. Q. Dai, Y. Y. Wang, J. F. Zhang, Ultrashort self-similar solutions of the cubic-quintic Nonlinear Schrödinger equation with distributed coefficients in the inhomogeneous fiber, J. Phys. A: Math. Theor. 44 (2011) 155203.

[12] Q. F. Zhang, H. Z. Li, L. M. Wu, J. Gao, Influence of dispersion distribution on the propagation and compression of self-similar optical beam, Eur. Phys. J. D 73 (2019) 26. 
[13] Shally Loomba, M. S. Mani Rajan, K. Gupta, H. Kaur, C. N. Kumar, Nonlinear tunneling of optical similaritons in a tapered graded-index nonlinear waveguide, Opt. Commun. 324 (2014) 286-295.

[14] H.Triki, C. Bensalem, A. Biswas, S. Khan, Q. Zhou, S. Adesanya, S. P. Moshokoa, M. Belic, Self-similar optical solitons with continuouswave background in a quadratic-cubic non-centrosymmetric waveguide, Opt. Commun. 437 (2019) 392-398.

[15] C. Mei, K. Wang, J. H. Yuan, Z. Kang, X. T. Zhang, B. B. Yan, X. Z. Sang, Q. Wu, X. Zhou, C. X. Yu, G. Farrell, Self-Similar Propagation and Compression of the Parabolic Pulse in Silicon Waveguide, J. Lightwave Technol. 37(2019) 1990-1999.

[16] S. A. Prakash, V. Malathi, M. S. Mani Rajan, Shally Loomba, Controllable pulse width of bright similaritons in a tapered graded index diffraction decreasing waveguide, Chaos 26 (2016) 033115.

[17] Ritu Pal, Shally Loomba, C. N. Kumar, Chirped self-similar waves for quadratic-cubic nonlinear Schrödinger equation. Ann. Phys. 387 (2017) 213-221.

[18] K. Subramanian, T. Alagesan, A. Mahalingam, M. S. Mani Rajan, Propagation properties of optical soliton in an erbium-doped tapered parabolic index nonlinear fiber: soliton control, Nonlinear Dyn. 87 (2017) 1575-1587.

[19] P. Wang, L. Feng, T. Shang, L. X. Guo, G. H. Cheng, Y. J. Du, Analytical soliton solutions for the cubic-quintic nonlinear Schrödinger equation with Raman effect in the nonuniform management systems, Nonlinear Dyn. 79 (2015) 387-395.

[20] X. Y. Xie, Z.H. Yan, Soliton collisions for the Kundu-Eckhaus equation with variable coefficients in an optical fiber, Appl. Math. Lett. 80 (2018) 48-53.

[21] A. Choudhuri, H. Triki, K. Porsezian, Self-similar localized pulses for the nonlinear Schrödinger equation with distributed cubic-quintic nonlinearity, Phys.Rev.A. 94 (2016) 063814.

[22] Z. X. Liang, Z. D. Zhang, W. M. Liu, Dynamics of a Bright Soliton in Bose-Einstein Condensates with Time-Dependent Atomic Scattering Length in an Expulsive Parabolic Potential, Phys. Rev. Lett. 94 (2005) 050402.

[23] P. Wang, T. Shang, L. Feng, Y. J. Du, Solitons for the cubic-quintic nonlinear Schrödinger equation with Raman effect in nonlinear optics, Opt. Quant Electron. 46 (2014) 1117-1126.

[24] R. Hirota, The Direct Method in Soliton Theory, Cambridge Univ, Press, Cambridge, 2004.

[25] A. C. Newell, Nonlinear tunneling, J. Math. Phys. 19 (1978) 1126-1133.

[26] V. N. Serkin, V. M. Chapela, J. T. Percino, L. Belyaeva, Nonlinear tunneling of temporal and spatial optical solitons through organic thin films and polymeric waveguides, Opt. Commun. 192 (2001) 237-244.

[27] V. N. Serkin, T. L. Belyaeva, High-energy optical Schrödinger solitons, J ETP Letters 74 (2001) 573-577.

[28] G. Y. Yang, R. Y. Hao, L. Li, Z. H. Li, G. S. Zhou, Cascade compression induced by nonlinear barriers in propagation of optical solitons, Opt. Commun. 260 (2006) 282-287.

[29] R. C. Yang, X. L. Wu, Spatial soliton tunneling, compression and splitting, Opt. Express 16 (2008) 17759.

[30] W. P. Zhong, M. R. Belić, Soliton Tunneling in the nonlinear Schrödinger equation with variable coefficients and an external harmonic potential, Phys. Rev. E 81 (2010) 056604.

[31] T. Marest, F. Braud, M. Conforti, S. Wabnitz, A. Mussot, A. Kudlinski, Longitudinal soliton tunneling in optical fiber, Opt. Lett. 42 (2017) 2350.

[32] P. Sprenger, M. A. Hoefer, G. A. El, Hydrodynamic optical soliton tunneling, Phys. Rev. E 97 (2018) 032218.

[33] J. F. Wang, L. Li, S. T. Jia, Nonlinear tunneling of optical similaritons in nonlinear waveguides, J. Opt. Soc. Am. B 25 (2008) $1254-1260$.

[34] C. Q. Dai, Y. Y. Wang, J. F. Zhang, Nonlinear similariton tunneling effect in the birefringent fiber, Opt. Express 18 (2010) 17548.

[35] H. P. Jia, R. C. Yang, C. Q. Dai, Y. Y. Guo, Spectral characteristics of periodic tunneling of chirped soliton in nonautonomous system with external potentials, J. Mod. Opt. 66 (2019) 665-673.

[36] M. S. Mani Rajan, A. Mahalingam, A. Uthayakumar, Nonlinear tunneling of nonautonomous optical solitons in combined nonlinear Schrödinger and Maxwell-Bloch systems, J. Opt.14 (2012) 105204-105207. 
[37] A. Mahalingam, M. S. Mani Rajan, Influence of generalized external potentials on nonlinear tunneling of nonautonomous solitons: Soliton Management, Opt. Fiber Technol. 25 (2015) 44-50.

[38] Shally Loomba, M. S. Mani Rajan, Rama Gupta; Harleen Kaur; C.N.Kumar, Nonlinear tunneling of optical similaritons in a tapered gradedindex nonlinear waveguide, Opt. Commun. 324 (2014) 286-295. 